\title{
DACH Polymer Platinate AP5346
}

National Cancer Institute

\section{Source}

National Cancer Institute. DACH Polymer Platinate AP5346. NCI Thesaurus. Code C64784.

A low molecular weight polymer-conjug ated platinum complex with potential antineoplastic activity. This polymer drug delivery system consists of cytotoxic diaminocyclohexane (DACH)-platinum (Pt) coupled to a water-soluble biocompatible hydroxypropylmethacrylamide (HPMA) copolymer via a pH sensitive linker. Due to decreased $\mathrm{pH}$ at the tumor site, the linker is cleaved and the chelated active moiety DACH-Pt is released in tumor cells. DACH-Pt alkylates macromolecules and causes both inter- and intra-strand platinum-DNA crosslinks, which impede DNA replication and transcription, thereby resulting in cell-cycle independent cytotoxicity. The HPMA-based drug delivery system enhances the concentration of DACH-Pt at the tumor site and prolongs the half life of the agent, thereby increasing exposure and efficacy at the target tumor sites while minimizing side effects in normal tissues. 